\title{
GREIT: a unified approach to 2D linear EIT reconstruction of lung images
}

\author{
Andy Adler ${ }^{1}$, John H. Arnold ${ }^{2}$, Richard Bayford ${ }^{3}$, Andrea \\ Borsic $^{4}$, Brian Brown ${ }^{5}$, Paul Dixon ${ }^{6}$, Theo J.C. Faes ${ }^{7}$, Inéz \\ Frerichs $^{8}$, Hervé Gagnon ${ }^{9}$, Yvo Gärber ${ }^{10}$, Bartłomiej \\ Grychtol $^{11}$, Günter Hahn ${ }^{12}$, William R B Lionheart ${ }^{13}$, Anjum \\ Malik $^{14}$, Robert P. Patterson ${ }^{15}$, Janet Stocks ${ }^{16}$, Andrew \\ Tizzard $^{3}$, Norbert Weiler ${ }^{8}$, Gerhard K. Wolf ${ }^{2}$
}

${ }^{1}$ Systems and Computer Engineering, Carleton University, Ottawa, Canada

${ }^{2}$ Division of Critical Care Medicine, Department of Anesthesia, Children's Hospital Boston, Harvard Medical School, Boston, MA, USA

${ }^{3}$ Department of Natural Sciences, Middlesex University, London, UK

${ }^{4}$ School of Engineering, Dartmouth College, Hanover, NH, USA

${ }^{5}$ Medical Physics, University of Sheffield, UK

${ }^{6}$ Cardinal Health Care, London, UK

${ }^{7}$ Department of Physics and Medical Technology, V.U. university medical center, Amsterdam, Netherlands

${ }^{8}$ Department of Anaesthesiology and Intensive Care Medicine, University of Schleswig-Holstein, Kiel, Germany

${ }^{9}$ Département de génie électrique, École Polytechnique de Montréal, Canada

${ }^{10}$ Dräger Medical, Lübeck, Germany

${ }^{11}$ University of Strathclyde, Glasgow, UK

${ }^{12}$ Department of Anaesthesiological Research, University of Göttingen, Germany

${ }^{13}$ School of Mathematics, University of Manchester, UK

${ }^{14}$ Maltron International, Rayleigh, UK

${ }^{15}$ Department of Biomedical Engineering, University of Minnesota, Minneapolis, MN, USA

${ }^{16}$ Institute of Child Health, University College London, UK

\begin{abstract}
Electrical Impedance Tomography (EIT) is an attractive method for clinically monitoring patients during mechanical ventilation, because it can provide a non-invasive continuous image of pulmonary impedance which indicates the distribution of ventilation. However, most clinical and physiological research in lung EIT is done using older and proprietary algorithms; this is an obstacle to interpretation of EIT images because the reconstructed images are not well characterized. To address this issue, we are developing a consensus linear reconstruction algorithm for lung EIT, called GREIT (Graz consensus Reconstruction algorithm for EIT). This paper describes the unified approach to linear image reconstruction developed for GREIT. The framework for the linear reconstruction algorithm consists of: 1) detailed finite element models of a representative adult and neonatal thorax; 2) consensus on the performance figures of merit for EIT image reconstruction; and 3) a systematic approach to optimize a linear reconstruction matrix to desired performance measures. Consensus figures of merit, in order of importance, are: a) uniform amplitude response,
\end{abstract}


b) small and uniform position error, c) small ringing artefacts, d) uniform resolution, e) limited shape deformation, and f) high resolution. Such figures of merit must be attained while maintaining small noise amplification and small sensitivity to electrode and boundary movement. This approach represents the consensus of a large and representative group of experts in EIT algorithm design and clinical applications for pulmonary monitoring. All software and data to implement and test the algorithm has been made available under an open source license which allows free research and commercial use.

Keywords: Electrical Impedance Tomography, Lung Function Imaging, Image Reconstruction,

\section{Introduction}

Electrical Impedance Tomography (EIT) measures conductivity changes within a body, by measuring the voltages at electrodes on the body surface caused when a number of different currents are passed through the body. One of the most promising applications of EIT is for description of physiologic events in the thorax, since the thorax contains several organs which undergo large changes in conductivity during normal functioning. Indeed, lung function measurement was among the first physiological applications of this technology (Barber and Brown 1984). EIT is unique in that it is able to non-invasively measure chest impedance to give a continuous image of the distribution of ventilation. Based on these advantages, there is significant interest in EIT to monitor patients with respiratory compromise (Frerichs et al 2003, Victorino et al 2004, Wolf et al 2007).

One limitation is that most clinical and physiological research on lung EIT is being done using proprietary variants of older image reconstruction algorithms, such as the backprojection algorithm as implemented in the Sheffield (Brown and Seagar 1987) or Göttingen (Hahn et al 2002) EIT systems. The algorithms, although very successful for the time, do not incorporate advances that have been made over the last 20 years. The reconstructed images show spatial non-uniformity in image amplitude, position and resolution, which make interpretation of regional ventilation difficult and error prone. For example, because of position errors, changes near the skin can appear as contrasts in the lung regions. Many other approaches to the reconstruction of EIT images have been proposed, incorporating many advances (Lionheart, 2004); however, such approaches have not been widely used clinically and experimentally because there was a lack of an agreement on which approaches were best (and how they could be combined).

To address this issue, we are developing a consensus linear reconstruction algorithm for lung EIT, called GREIT (Graz consensus Reconstruction algorithm for EIT), since early discussions took place at the 2007 ICEBI conference in Graz, Austria. Our aim is to develop a standard which has broad agreement from experts in the mathematical, engineering, physiological, and clinical EIT communities. Such an approach is feasible because there is general consensus amongst experts in lung EIT applications on the "ingredients" that should be part of a robust and high performance linear algorithm 
for 2D EIT of the lungs. This paper describes the unified approach to linear image reconstruction developed for GREIT, and the tools and evaluation methodology for clinical and experimental testing of the algorithm.

The current work is limited to the reconstruction algorithm. At this stage, we do not propose calibration tests, data formats or phantoms, standards for image interpretation or EIT based lung parameters; we do not feel there is sufficient experience yet to reach consensus in these areas. It is important to clarify that there is no financial goal to the development of this algorithm. All developed algorithms, software models and simulation and experimental test data used in this algorithm have been made available under an open source license as part of the open source EIDORS distribution (Adler and Lionheart 2006); this license permits royalty free use in both research and commercial applications. Additionally, due to space constraints in this paper, only a limited set of results could be included. The complete algorithms, results, and source data are available on the internet at eidors.org/GREIT.

The following specifications have been defined for GREIT:

- single ring electrode configurations with Sheffield-type EIT systems, using adjacent current injection and measurement.

- linear (i.e. real-time) reconstruction of a 2D conductivity change image, based on a 3D forward model.

- quantitative difference reconstructions for which units can be assigned to EIT images.

- reconstruction onto a $32 \times 32$ pixel array for a single ring of 16, 12 and 8 electrodes, for the shapes: a) neonatal chest, b) male and female adult chest, and c) cylindrical tank.

These specifications represent, in our opinion, the limits to areas sufficiently well understood in the EIT community to reach consensus. We do not suggest that such limits are ideal; in fact, we actively encourage work to overcome them, such as the single ring placement of electrodes and the consequent lack of 3D image information.

\section{Methods: performance figures of merit}

In this section, we elaborate a set of criteria which characterize the performance of an ideal reconstruction algorithm. Clearly, there is an inherent trade-off between the criteria, such that it is not possible to simultaneously optimize all measures. Instead, we proceed as follows. First, we establish the desired performance measures of an ideal algorithm; subsequently, we describe a unified methodology to calculate a reconstruction matrix which is an optimized compromise of criteria weighted by the consensus importance of each.

We consider an EIT system using the following notation. Using $n_{E}$ electrodes, $n_{E}$ current stimulation patterns are sequentially applied and $n_{V}$ differential voltage measurements are made in parallel for each stimulation. For an adjacent drive EIT 
system, voltages are typically not measured at driven electrodes, and $n_{V}=n_{E}-3$. Each data frame measures a vector, $\mathbf{v} \in \mathbb{R}^{n_{M}}$, of $n_{M}=n_{E} n_{V}$ data points (some of which are redundant if the medium is not changing). Difference EIT calculates difference data $\mathbf{y}$, $\left([\mathbf{y}]_{i}=[\mathbf{v}]_{i}-\left[\mathbf{v}_{r}\right]_{i}\right)$; or the normalized difference data $\left.[\mathbf{y}]_{i}=\left([\mathbf{v}]_{i}-\left[\mathbf{v}_{r}\right]_{i}\right) /\left[\mathbf{v}_{r}\right]_{i}\right)$, where $\mathbf{v}_{r}$ is a reference set of measurements corresponding to the background conductivity distribution, $\boldsymbol{\sigma}_{r}$. To improve its precision, $\mathbf{v}_{r}$ is typically averaged over many data frames; such ensemble averaging reduces random noise, and we assume that $\mathbf{v}_{r}$ is noise free.

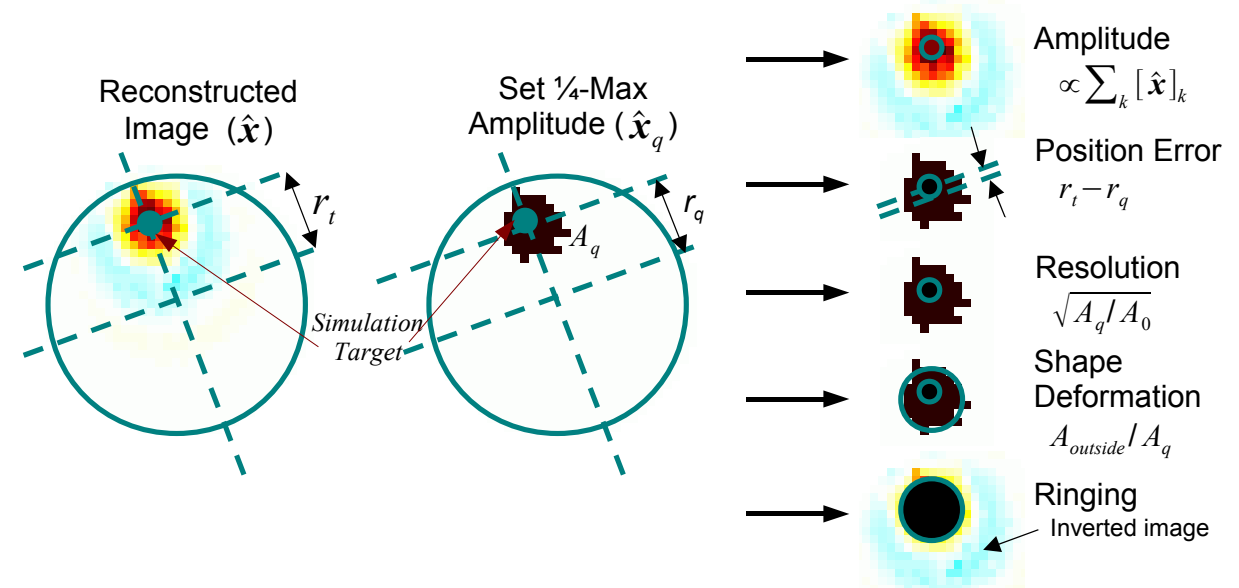

Figure 1. Performance figures of merit for evaluation of GREIT images. Based on a reconstructed image $(\hat{\mathbf{x}}$, left $)$, an image $\left(\hat{\mathbf{x}}_{q}\right.$, centre $)$ is constructed of all image pixels which exceed $\frac{1}{4}$ the maximum amplitude. From these images, figures of merit at right are calculated as described.

An outline of the calculation of performance figures of merit is given in Fig. 1. Given a vector of EIT difference or normalized difference data, $\mathbf{y}$ (length $n_{E} n_{V}$ ), we calculate a reconstructed EIT image $\hat{\mathbf{x}}=\mathbf{R} \mathbf{y}$ based on an EIT linear reconstruction algorithm represented as a matrix R. $\hat{\mathbf{x}}$ is a column vector representing the $32 \times 32$ pixel grid of the images.

We define figures of merit based on small "point" conductivity changes, where small indicates a diameter of less than $5 \%$ of the medium diameter, and thus much smaller than the inherent resolution of EIT with 16 electrodes. To evaluate this image, we first calculate a $\frac{1}{4}$-amplitude set, $\hat{\mathbf{x}}_{q}$, which contains all image pixels $[\hat{\mathbf{x}}]_{i}$ greater than $\frac{1}{4}$ of the maximum amplitude:

$$
\left[\hat{\mathbf{x}}_{q}\right]_{i}= \begin{cases}1 & \text { if }[\hat{\mathbf{x}}]_{i} \geq \frac{1}{4} \max (\hat{\mathbf{x}}) \\ 0 & \text { otherwise }\end{cases}
$$

For targets with a conductivity decrease, the definition of the $\frac{1}{4}$-amplitude set is inverted. A threshold of $\frac{1}{4}$ represents a compromise which detects most of the visually significant effects; however, the exact value of the threshold has little effect on the final algorithm parameters. The centre of gravity $(\mathrm{CoG})$ of $\hat{\mathbf{x}}$ and $\hat{\mathbf{x}}_{q}$ are calculated, and the distance from the CoG to the medium centre is then calculated, as $r_{t}$ and $r_{q}$ respectively.

Based on images of point targets, we define the following figures of merit: 
- Amplitude Response (AR) measures the ratio of image pixel amplitudes in the target to that in the reconstructed image. For a spherical target of volume $V_{t}$ in the electrode plane with conductivity $\sigma_{t}$ in a body of homogeneous reference conductivity $\sigma_{r}$

$$
\mathrm{AR}=\frac{\sum_{k}[\hat{\mathbf{x}}]_{k}}{V_{t} \frac{\Delta \sigma}{\sigma_{r}}}
$$

where $\Delta \sigma=\sigma_{t}-\sigma_{r}$.

AR allows units to be assigned to the reconstructed conductivities. For this, we require that reconstruction matrices, $\mathbf{R}$, be scaled such that $\mathrm{AR}=1$ for a small spherical target with $\Delta \sigma / \sigma_{r} \approx 1$ in the centre of the body. With this normalization, reconstructed EIT images have units as follows:

- Normalized Difference EIT: in this case, measurements y are unitless, and the EIT image will be in units of fractional conductivity change, $\Delta \sigma / \sigma_{r}$.

- Difference EIT: in this case, measurements $\mathbf{y}$ must be in units of transfer conductance $\left(\Omega^{-1}\right)$, and the EIT image will be in units of conductivity change (in $\Omega^{-1} \cdot \mathrm{m}$ ) scaled by the ratio or the measurement geometry to the FEM geometry.

Desired behaviour: AR should be constant for any target position. We consider constant AR to be the most important figure of merit, since, without constant amplitude response, the same volume of air in different parts of the lung will contribute differently to the image, introducing serious difficulties in image interpretation.

- Position Error (PE): measures the extent to which reconstructed images faithfully represent the position of the image target. Based on the target position, $r_{t}$, and the CoG of $\hat{\mathbf{x}}_{q}, r_{q}$, we define:

$$
\mathrm{PE}=r_{t}-r_{q}
$$

where positive values of PE indicate reconstructed images "pushed" to the medium centre. This measure is similar to that of Adler and Guardo (1996).

Desired behaviour: PE should be small and show small variability for targets at different radial positions. We consider small and constant PE to be the second most important figure of merit. If $\mathrm{PE}$ is variable, interpretation of a distribution of air in the lungs becomes unreliable. Sheffield backprojection has large PE near the electrodes, and this has resulted in cases where changes at the electrodes are misinterpreted as being inside the body.

- Resolution (RES): measures the size of reconstructed targets as a fraction of the medium; this is equivalent to a measure of point spread function (PSF) size.

$$
\mathrm{RES}=\sqrt{\frac{A_{q}}{A_{0}}}
$$

where $A_{q}=\sum_{k}\left[\hat{\mathbf{x}}_{q}\right]_{k}$, the number of pixels in $\hat{\mathbf{x}}_{q}$ and $A_{0}$ is the area (in pixels) of the entire reconstructed medium. The square root is used so that RES measures 
radius ratios rather than area ratios. This measure is modelled on the one proposed by Wheeler et al (2002).

Desired behaviour: RES should be uniform and small, in order to more accurately represent shape of the target conductivity distribution. We consider uniformity of RES to be the fourth most important figure of merit, but small size of RES to be less important (sixth). A non-uniform RES can result in an incorrect reconstructed position of a larger target. Low RES serves primarily to distinguish nearby targets. Since EIT is understood to be a low resolution medium, this application is less important.

- Shape Deformation (SD): Reconstruction algorithms typically create circular images for targets in the centre, but often display strangely shaped artefacts for targets near the medium boundary. The Sheffield backprojection algorithm tends to show "streak" artefacts in this case. SD measures the fraction of the reconstructed $\frac{1}{4}$-Amplitude set which does not fit within a circle of equal area.

$$
\mathrm{SD}=\sum_{k \notin C}\left[\hat{\mathbf{x}}_{q}\right]_{k} / \sum_{k}\left[\hat{\mathbf{x}}_{q}\right]_{k}
$$

where $C$ is a circle centred at the $\mathrm{CoG}$ of $\hat{\mathbf{x}}_{q}$ with an equivalent area to $A_{q}$. This figure is similar to that of Oh et al (2007).

Desired behaviour: SD should be low and uniform. Large SD may result in incorrect interpretation of images, although this effect is less important than other artefacts. We consider SD to be the fifth most important figure of merit.

- Ringing (RNG): measures whether reconstructed images show areas of opposite sign surrounding the main reconstructed target area. Fig. 1 shows an arc of ringing around the image centre. Ringing is typical of linear filters; for secondorder systems the effect may also be called "overshoot". RNG measures the ratio of image amplitude of the opposite sign outside circle $C$ to image amplitude within C.

$$
\mathrm{RNG}=\left(\sum_{k \notin C \&[\hat{\mathbf{x}}]_{k}<0}[\hat{\mathbf{x}}]_{k}\right) /\left(\sum_{k \in C}[\hat{\mathbf{x}}]_{k}\right)
$$

Desired behaviour: RNG should be low and uniform. Overshoot may easily result in incorrect interpretation. For example, the negative ringing between nonconductive lungs will produce a conductive pattern which may be misinterpreted as a heart. We consider RNG to be the third most important figure of merit.

- Noise Amplification (NF): measures the extent to which random measurement noise is amplified in the reconstructed images. Noise amplification is measured by the noise figure (NF) as illustrated in Fig. 2, based on the definition by Adler and Guardo (1996). The NF is the ratio of the output to input signal to noise ratio $(\mathrm{SNR})$ for a filter. We define $\mathrm{SNR}=\frac{\text { mean } \mid \text { signal } \mid}{\mathrm{std} \text { noise }}$ in terms of image amplitude, rather than in terms of image energy, since linear image reconstruction tends to 


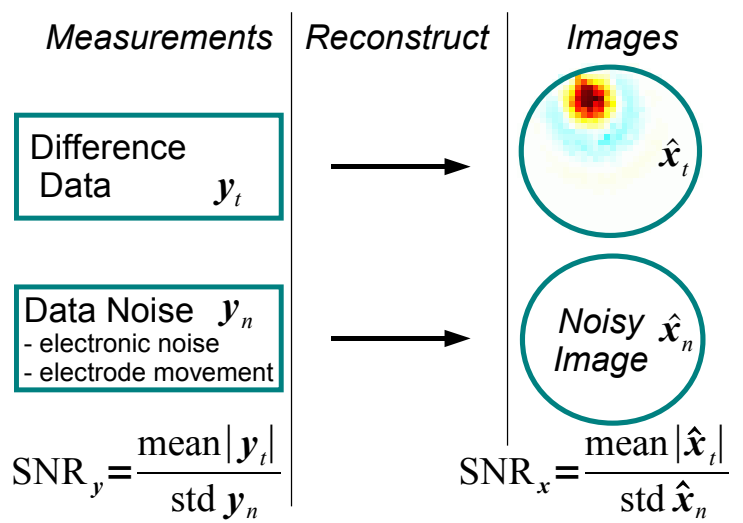

Figure 2. Schematic representation of the Noise Figure (NF) parameter. NF represents the amplification of noise through the reconstruction process as the ratio of $\mathrm{SNR}_{x}$ to $\mathrm{SNR}_{y}$.

conserve amplitude rather than energy. Thus

$$
\mathrm{NF}=\frac{E\left[\text { mean }\left|\hat{\mathbf{x}}_{t}\right|\right] / E\left[\operatorname{std} \hat{\mathbf{x}}_{n}\right]}{E\left[\text { mean }\left|\mathbf{y}_{t}\right|\right] / E\left[\operatorname{std} \mathbf{y}_{n}\right]}
$$

For regularized algorithms, the value of NF is normally set by selection of the value of a hyperparameter. For GREIT, the value of NF is set by the weighting associated with the training noise. There is an inherent trade-off betneen good noise performance and fidelity to the other figures of merit.

Desired behaviour: NF should be low, and, ideally, should be tuned to the noise level present in the EIT hardware used. Several studies have looked into strategies to select appropriate noise performance (eg. Graham and Adler 2006). At this time, we do not have consensus on the best way to choose NF. Instead, we note that noise performance of Sheffield Backprojection ( $\mathrm{NF}=0.5$ in the medium center) has generally been considered to be satisfactory. Therefore, as an interim measure, we recommend $\mathrm{NF}=0.5$ for GREIT algorithms.

\section{Methods: Reconstruction algorithm framework}

This section describes the GREIT framework to calculate a linear image reconstruction matrix which is optimized to a set of performance requirements. This approach is based on experience with a large number of linear regularized EIT reconstruction approaches (such as Adler and Guardo 1996, Adler and Lionheart 2006, Barber and Brown 1988, Cheney et al 1990, Cohen-Bacrie et al 1997, Polydorides and Lionheart 2002, Soleimani et al 2006, Vauhkonen et al 1998). The key difference is that this framework is based on a set of performance requirements, whereas previous reconstruction algorithms are based directly on the underlying mathematical models and do not express the performance requirements explicitly. The value of this approach is that it allows these performance requirements (established in our case via consensus) to be directly encoded into the reconstruction algorithm. 
The body under investigation is modelled using a finite element model (FEM) which discretizes the conductivity onto $n_{N}$ piecewise smooth elements, represented by a vector $\boldsymbol{\sigma} \in \mathbb{R}^{n_{N}}$ (To clarify use of symbols, $\boldsymbol{\sigma}$ represents a vector conductivity distribution, while $\sigma$ is the standard deviation). Difference EIT calculates a vector of conductivity change, $\mathbf{x}=\boldsymbol{\sigma}-\boldsymbol{\sigma}_{r}$ between the present, $\boldsymbol{\sigma}$, and the reference, $\boldsymbol{\sigma}_{r}$, conductivity distributions. $\mathbf{x}$ may also represent a parameter $\log \frac{\boldsymbol{\sigma}}{\boldsymbol{\sigma}_{r}}$, which matches the conductivity change in the linear approximation.

Image reconstruction in EIT is a non-linear problem; however, linearized approximations have proved to be very useful. This is largely because the measurement noise and geometric uncertainty present in clinical and experimental EIT data are sufficiently large to prevent stable results with iterative algorithms. Linear algorithms also have the benefit of producing images in which the effects of data artefacts can be more readily identified. Finally, linear reconstruction can be implemented as a fast matrix multiplication. We represent linear EIT image reconstruction as a matrix, $\mathbf{R} \in \mathbb{R}^{n_{N} \times n_{M}}$ which maps measurements $\mathbf{y}$ to a reconstructed image $\hat{\mathbf{x}}$ :

$$
\hat{\mathbf{x}}=\mathbf{R y}
$$

\subsection{Sheffield Backprojection}

The backprojection algorithm was developed by Barber and Brown (1984) for the original Sheffield EIT system, and has seen numerous variants and improvements (Barber, 1989). While the original technique was based on an analogy to the backprojection algorithm for CT, this model was clearly inadequate, because electric current propagates diffusely, and thus differently to X-ray photons. Therefore, improvements were developed to the backprojection algorithm (e.g. Barber and Brown 1988). Such strategies can be shown to regularize the reconstructed images. A good mathematical characterization is given by Santosa and Vogelius (1990).

While many variants of Sheffield backprojection exist, clinical and experimental EIT has largely used only the one which was distributed in the Sheffield Mark I EIT system (Brown and Seagar 1987). The Göttingen Goe MF II EIT system (Hahn et al 2002) has also been widely used for such clinical and experimental work, and uses a very similar reconstruction algorithm.

Unfortunately, it appears that the exact formulation of this algorithm has been lost. To regenerate it, we proceeded as follows. Since image reconstruction is linear, there exists a mapping which can explain the transformation of measurements into images. From a Sheffield Mark I device, we obtained a large set of measurements, $\mathbf{y}_{k}$, and the associated reconstructed images, $\hat{\mathbf{x}}_{k}$, and formed matrices $\mathbf{Y}=\left[\mathbf{y}_{1}, \cdots, \mathbf{y}_{k}, \cdots\right]$ and $\mathbf{X}=\left[\hat{\mathbf{x}}_{1}, \cdots, \hat{\mathbf{x}}_{k}, \cdots\right]$ by concatenation. The Sheffield backprojection matrix, was calculated as the least squared fit to $\mathbf{Y} \mathbf{R}_{S B P}=\mathbf{X}$. In order to permit testing of EIT data against this algorithm, it has been made available online eidors.org/data_contrib/db_backproj_matrix under the following license: "This matrix is copyright DC Barber and BH Brown at University of Sheffield. It may be 
used free of charge for research and non-commercial purposes. Commercial applications require a license from the University of Sheffield."

\subsection{One step linear Gauss-Newton solvers}

In this section, we elaborate the Gauss-Newton (GN) EIT reconstruction approaches, which have been widely used in EIT since the late 1980's (Yorkey et al 1987, Cheney et al 1990). This approach allows use of sophisticated regularized models of the EIT inverse problem, able to represent this solution as a linear reconstruction matrix, which can then allow rapid, real-time imaging. For small variations around the reference conductivity $\boldsymbol{\sigma}_{r}$, the relationship between $\mathbf{x}$ and $\mathbf{y}$ can be linearized (giving the difference EIT forward model):

$$
\mathbf{y}=\mathbf{J} \mathbf{x}+\mathbf{n}
$$

where $\mathbf{J} \in \mathbb{R}^{n_{M} \times n_{N}}$ is the Jacobian or sensitivity matrix and $\mathbf{n} \in \mathbb{R}^{n_{M}}$ is the measurement noise, which is assumed to be uncorrelated white Gaussian. $\mathbf{J}$ is calculated from the FEM as $[\mathbf{J}]_{i j}=\left.\frac{\partial[\mathbf{y}]_{i}}{\partial[\mathbf{X}]_{j}}\right|_{\boldsymbol{\sigma}_{r}}$, and depends on the FEM, the current injection patterns, the reference conductivity, and the electrode models. This system is underdetermined since $n_{N}>n_{M}$. Regularization techniques are required in order to calculate a conductivity change estimate, $\hat{\mathbf{x}}$, which is both faithful to the measurements, $\mathbf{y}$, and to a priori constraints on a "reasonable" image.

The GN inverse problem seeks to calculate a solution, $\hat{\mathbf{x}}$, to the EIT inverse problem in terms of a generalized Tikhonov regularization expressed as the minimum of a sum of quadratic norms

$$
\|\mathbf{y}-\mathbf{J} \hat{\mathbf{x}}\|_{\Sigma_{n}^{-1}}^{2}+\left\|\mathbf{x}-\mathbf{x}^{\circ}\right\|_{\Sigma_{x}^{-1}}^{2}
$$

where $\mathbf{x}^{\circ}$ represents the expected value of element conductivity changes, which is zero for difference EIT. $\boldsymbol{\Sigma}_{n} \in \mathbb{R}^{n_{M} \times n_{M}}$ is the covariance matrix of the measurement noise $\mathbf{n}$. Since $\mathbf{n}$ is uncorrelated, $\boldsymbol{\Sigma}_{n}$ is a diagonal matrix with $\left[\boldsymbol{\Sigma}_{n}\right]_{i, i}=\sigma_{i}^{2}$, where $\sigma_{i}^{2}$ is the noise variance at measurement $i . \Sigma_{x} \in \mathbb{R}^{n_{N} \times n_{N}}$ is the expected image covariance.

It is worth noting that many other regularized approaches to EIT have been used, such as the TSVD (truncated singular value decomposition; Bayford et al 2008) and SIRT (simultaneous iterative reconstruction technique; Hahn et al 2006). All linear approaches are structurally similar; however, the formulation in (10) explicitly exposes the selection of the image reconstruction parameters.

Typically, covariance matrices $\boldsymbol{\Sigma}_{n}$ and $\boldsymbol{\Sigma}_{x}$ are not calculated directly, but are modelled heuristically from a priori considerations as $\mathbf{V}=\sigma_{n}^{2} \boldsymbol{\Sigma}_{n}$ and $\mathbf{P}=\sigma_{x}^{2} \boldsymbol{\Sigma}_{x}$, where $\sigma_{n}$ is the average measurement noise amplitude and $\sigma_{x}$ is the a priori amplitude of conductivity change. V models the measurement accuracy. For uncorrelated noise, each diagonal element is proportional to the signal to noise ratio. For difference EIT with identical channels, $\mathbf{V}=\mathbf{I}$. The regularization matrix $\mathbf{P}$ may be understood to model the likelihood of image elements and their interactions. 
By solving (10), a linearized, one-step inverse solution is obtained as

$$
\begin{aligned}
\hat{\mathbf{x}} & =\left(\mathbf{J}^{T} \frac{1}{\sigma_{n}^{2}} \mathbf{V}^{-1} \mathbf{J}+\frac{1}{\sigma_{x}^{2}} \mathbf{P}^{-1}\right)^{-1} \mathbf{J}^{T} \frac{1}{\sigma_{n}^{2}} \mathbf{V}^{-1} \mathbf{y} \\
& =\left(\mathbf{J}^{T} \mathbf{V}^{-1} \mathbf{J}+\lambda^{2} \mathbf{P}^{-1}\right)^{-1} \mathbf{J}^{T} \mathbf{V}^{-1} \mathbf{y}
\end{aligned}
$$

where parameter $\lambda=\sigma_{n} / \sigma_{x}$ is often called the "regularization hyperparameter" and controls the trade-off between resolution and noise attenuation in the reconstructed image. The matrix, $\mathbf{R}=\left(\mathbf{J}^{T} \mathbf{V}^{-1} \mathbf{J}+\lambda^{2} \mathbf{P}^{-1}\right)^{-1} \mathbf{J}^{T} \mathbf{V}^{-1}$ is the linear, one-step inverse. In (11), the term in the inverse is of size $n_{N} \times n_{N}$. To save computational time, and improve inverse accuracy and stability, matrix $\mathbf{R}$ may be rewritten using the data form, or Wiener filter form as:

$$
\mathbf{R}=\mathbf{P} \mathbf{J}^{T}\left(\mathbf{J P J}^{T}+\lambda^{2} \mathbf{V}\right)^{-1}
$$

In (12), the size of the term in the inverse is reduced to $n_{M} \times n_{M}$.

If image elements are assumed to be independent with identical variance, $\mathbf{P}$ becomes an identity matrix I and (11) uses zeroth-order Tikhonov regularization. For EIT, such solutions tend to push reconstructed noise toward the boundary, since the measured data are much more sensitive to boundary image elements. Instead, $\mathbf{P}$ may be scaled

with the sensitivity of each element, so that each diagonal element $i,\left[\mathbf{P}^{-1}\right]_{i, i}=\left[\mathbf{J}^{T} \mathbf{J}\right]_{i, i}$. This is the NOSER prior of Cheney et al (1990). Many other prior matrices have been proposed: to model image smoothness as a penalty for non-smooth image regions, $\mathbf{P}$ may be set to the discrete Laplacian filter (Vauhkonen et al 1998), a discrete high pass Gaussian filter (Adler and Guardo 1996), or based on variance uniformization constraints (Cohen-Bacrie et al 1997). In this paper, we compare results from the GREIT approach with those of GN image reconstruction using the NOSER prior. This algorithm is representative of a broad group of structurally similar algorithms that have been widely implemented in EIT; it is considered to generally perform well.

\subsection{GREIT image reconstruction approach}

In this section, we elaborate the GREIT procedure from which the reconstruction matrix, $\mathbf{R}$ is calculated. The procedure depends on definition of a forward model, a noise model, and desired performance metrics.

3.3.1. Forward model A forward model allows calculation of EIT measurement data $\mathbf{y}^{(k)}$ from a conductivity change distribution $\mathbf{x}^{(k)}$. The model represents the details of the body geometry, the electrode size and contact impedance, and the reference conductivity, $\boldsymbol{\sigma}_{r}$, around which conductivity changes occur. In this paper, the forward problem is solved using a 3D FEM using the complete electrode model (Cheng et al 1989). However, it is worth noting that any forward model, physical or numerical, is suitable for this procedure.

One other requirement for the forward problem model is an estimate of the amplitude of conductivity changes $\mathbf{x}^{(k)}$ encountered in the modelled EIT application. 
Based on the forward model, conductivity changes, $\mathbf{x}$, result in a measurement variance $\sigma_{\mathbf{y}}^{2}=\operatorname{var} \mathbf{y}=E\left[\|\mathbf{y}\|^{2}\right]$. The mean $\mathbf{y}$ is assumed to be zero, which implies that positive and negative conductivity changes are equally likely. This assumption is reasonable for a general purpose EIT reconstruction algorithm, although specific EIT applications may not match it exactly. For lung images, we may calculate $\sigma_{\mathbf{y}}^{2}$ using either a simulation model of the amplitude of conductivity changes due to breathing, or from a sample of EIT measurements in representative applications.

3.3.2. Noise model A noise model allows calculation of representative noise (or undesired signal) samples in EIT measurements. For GREIT, we consider two sources of noise: electronic measurement noise, and electrode movement artefacts. In general, for each source of noise $n_{s}$, we have noise samples $\mathbf{y}_{n_{s}}^{(k)}$, and an estimate of the noise amplitude variance $\sigma_{n_{s}}^{2}=\operatorname{var} \mathbf{y}_{n_{s}}=E\left[\left\|\mathbf{y}_{n_{s}}\right\|^{2}\right]$. The mean noise is assumed to be zero in this formulation; if necessary, a non-zero noise mean should be accounted for by pre-processing of EIT measurements. Note that for normalized difference EIT, noise samples are normalized by the reference measurements by $[\mathbf{y}]_{i}=[\mathbf{n}]_{i} /\left[\mathbf{v}_{r}\right]_{i}$.

Electronic measurement noise is typically modelled to be uniform and Gaussian in EIT image reconstruction. This assumption is reasonable for a generic GREIT algorithm designed for general EIT reconstruction. However, for a specific EIT system, measurement noise is a function of the EIT hardware and patient connection. If different gain settings are used for each channel, these settings will alter the noise. Calibrated measurements of EIT noise show that it is non-Gaussian and varies between channels (Hahn et al 2008). We suggest GREIT algorithms be tuned to the hardware implementation. This may be implemented using a calibration protocol before the start of measurements, or by integrating a model of the hardware imperfections into the forward model (Hartinger et al 2007).

Electrode movement artefacts occur when the electrodes move, either with posture change or with chest movements due to breathing. Several reports have demonstrated the significant impact of such movements on EIT images (Adler et al 1996, Zhang and Patterson 2005, Coulombe et al 2005). In order to reduce the contribution of such movement on EIT reconstruction, Soleimani et al (2006) showed that it is possible to create an augmented forward model based on both the conductivity change and electrode movement, which resulted in reduced movement artefacts in the reconstructed images. In order to use this capability in GREIT, we specify that a set of "noise" measurements due to electrode movement be incorporated. This is currently implemented from deformations of the FEM (Gómez-Laberge and Adler 2008), but may be based on a calibration protocol in an implemented system.

3.3.3. Desired performance metrics. Based on the performance metrics defined in section 2, we create a training set of "desired images", $\tilde{\mathbf{x}}_{t}$. For training set member $(k)$, each image, $\tilde{\mathbf{x}}_{t}^{(k)}$, corresponds to the position of a conductivity target, $\mathbf{x}_{t}^{(k)}$, in the forward model, as illustrated in Fig. 3. The desired image, $\tilde{\mathbf{x}}_{t}$, is located at the same 


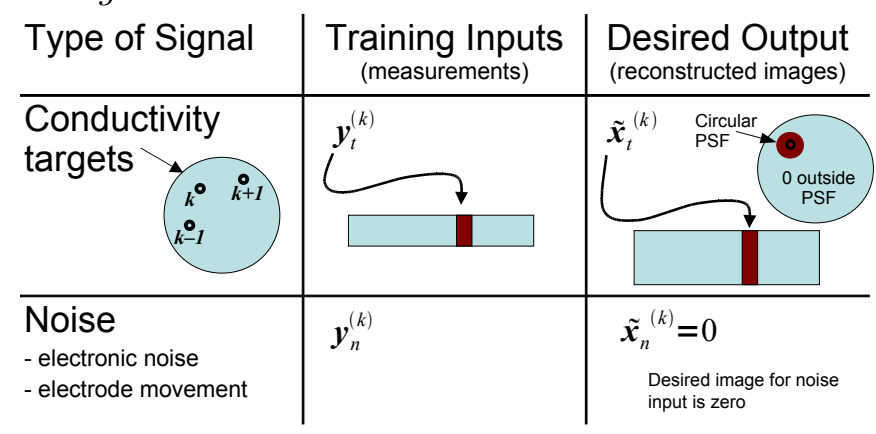

Figure 3. Training data for GREIT. Two sets of training data are used, based on conductivity targets and noise or artefact sources. For each type data, a set of training inputs (EIT measurements) and desired outputs (reconstructed images) are calculated. Rectangles represents a matrix in which each column $(k)$ represents a training sample. The circle represents corresponding desired image pattern.

centre as the conductivity target, $\mathbf{x}_{t}$. However, $\tilde{\mathbf{x}}_{t}$ is defined to be a circle with a circular area corresponding to the blurring inherent in EIT, while $\mathbf{x}_{t}$ is essentially a point target. It is this definition of a larger "desired image" which allows GREIT to achieve a more uniform resolution. Since most EIT reconstruction algorithms achieve better resolution near the boundary than in the medium centre, this definition of $\tilde{\mathbf{x}}_{t}$ forces a trade-off of boundary resolution for a lower resolution that can be achieved uniformly throughout the image. Note that it would possible to set $\tilde{\mathbf{x}}_{t}^{(k)}=\mathbf{x}_{t}^{(k)}$; however, this would be equivalent to a standard GN solution without tuning the algorithm to the performance metrics. We recommend, for a 16 electrode EIT system, that the diameter of $\tilde{\mathbf{x}}_{t}$ be $20 \%$ of the medium diameter.

Corresponding to each "desired image", is an image weighting $\mathbf{w}^{(k)}$ which represents the weight given to each pixel in $\tilde{\mathbf{x}}_{t}^{(k)}$. The weighting allows "tuning" of the relative importance of the performance metrics, as illustrated in Fig. 4. For each desired image, $\tilde{\mathbf{x}}_{t}^{(k)}$, there is an inner circular zone centred at the target position where the amplitude is required to be flat to meet the AR and PE performance metrics. There is another circular zone outside the boundary of the specified contrast, where the desired image is zero, to meet the RNG and SD performance metrics (illustrated as the circles on Fig. 4 bottom left). In these zones, $\mathbf{w}^{(k)}$ is large in order to provide a substantial penalty for images outside the specifications. Between the circles we have a "transition zone" in which the reponse gradually decreases from the specified amplitude to zero. In the transition zone, $\mathbf{w}^{(k)}$ is small to allow the reconstruction algorithm flexibility to meet the other specifications.

In addition to the conductivity targets, noise training samples, $\mathbf{y}_{n}$ are generated for data noise and electrode movement. For noise samples, the "desired image", $\tilde{\mathbf{x}}_{n}$, is clearly zero; we wish for noise input to produce no output. For these images, the weighting, $\mathbf{w}^{(k)}$ is uniform across the image area. The magnitude of $\mathbf{w}^{(k)}$ controls NF and the overall noise performance of GREIT. A large value of $\mathbf{w}^{(k)}$ results in a large penalty for image noise and a low NF, and vice vera. 


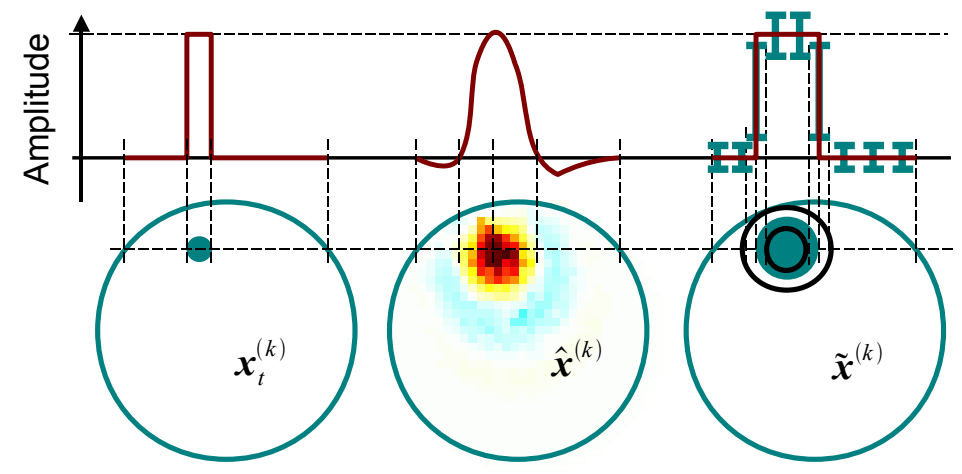

Figure 4. Illustration of the training data and weighting. left: Training target $\left(\mathbf{x}_{t}^{(k)}\right)$ centre: Reconstructed image $\left(\hat{\mathbf{x}}^{(k)}\right)$ right: Desired image $\left(\tilde{\mathbf{x}}_{t}^{(k)}\right)$ The bottom row shows the image, while the top row plots the amplitude across a row through the centre of the simulation target. $\tilde{\mathbf{x}}_{t}^{(k)}$ is larger than $\mathbf{x}_{t}^{(k)}$ in order to establish a uniform resolution. The weighting $\mathbf{w}^{(k)}$ is illustrated on the image of $\tilde{\mathbf{x}}_{t}^{(k)}$ by two circles. Inside the inner circle and outside the outer circle, $\mathbf{w}^{(k)}$ is larger, illustrated by the small error bars on the upper graph. Between the circles, in the "transition zone", $\mathbf{w}^{(k)}$ is smaller, illustrated by the larger error bars.

Based on the forward model, noise model, and desired performance metrics. we are able to formulate a set of training data and define the GREIT reconstruction matrix in terms of these data. We can show that for a linear reconstruction, this approach is equivalent to a generalized Tikhonov regularization (section 3.2) in which the prior distributions $\Sigma_{x}$ and $\Sigma_{n}$ correspond to the selection strategy for the training data (section 3.4). The GREIT reconstruction matrix $\mathbf{R}$ (later referred to as $\mathbf{R}_{G R}$ ) which best fits the requirements may be expressed as minimization of an error $\epsilon^{2}$

$$
\begin{aligned}
\epsilon^{2}= & \sum_{k}\left\|\tilde{\mathbf{x}}^{(k)}-\mathbf{R} \mathbf{y}^{(k)}\right\|_{\mathbf{W}^{(k)}}^{2} \\
= & \sum_{k} \sum_{i}\left(\left[\tilde{\mathbf{x}}^{(k)}\right]_{i}^{2}\left[\mathbf{w}^{(k)}\right]_{i}^{2}-2\left[\tilde{\mathbf{x}}^{(k)}\right]_{i}\left[\mathbf{w}^{(k)}\right]_{i}^{2}\left(\sum_{j} \mathbf{R}_{i j}\left[\mathbf{y}^{(k)}\right]_{j}\right)\right. \\
& \left.+\left[\mathbf{w}^{(k)}\right]_{i}^{2}\left(\sum_{j} \mathbf{R}_{i j}\left[\mathbf{y}^{(k)}\right]_{j}\right)^{2}\right)
\end{aligned}
$$

where the sum $k$ is over all training measurement and noise samples, and $\mathbf{W}^{(k)}=$ $\left(\operatorname{diag} \mathbf{w}^{(k)}\right)^{2}$, is a diagonal matrix representing the weighting corresponding to each measurement. The 2-norm is used as it allows a linear expression for, and faster computation of, $\mathbf{R}$; however, other norms may result in improved performance.

Based on (13), we develop an expression for $\mathbf{R}=\arg \min \epsilon^{2}$, by setting the matrix 
derivative $\frac{\partial \epsilon^{2}}{\partial \mathbf{R}_{i j}}$ to zero.

$$
\begin{aligned}
-\frac{1}{2} \frac{\partial \epsilon^{2}}{\partial \mathbf{R}_{i j}} & =\sum_{k}\left[\tilde{\mathbf{x}}^{(k)}\right]_{i}\left[\mathbf{w}^{(k)}\right]_{i}^{2}\left[\mathbf{y}^{(k)}\right]_{j}-\sum_{k}\left[\mathbf{w}^{(k)}\right]_{i}^{2}\left(\sum_{l} \mathbf{R}_{i l}\left[\mathbf{y}^{(k)}\right]_{l}\right)=0 \\
& =\sum_{k}\left[\tilde{\mathbf{x}}^{(k)}\right]_{i}\left[\mathbf{w}^{(k)}\right]_{i}^{2}\left[\mathbf{y}^{(k)}\right]_{j}-\sum_{l} \mathbf{R}_{i l}\left(\sum_{k}\left[\mathbf{y}^{(k)}\right]_{l}\left[\mathbf{w}^{(k)}\right]_{i}^{2}\left[\mathbf{y}^{(k)}\right]_{j}\right) \\
& =\mathbf{A}_{i j}-\sum_{l} \mathbf{R}_{i l} \mathbf{B}_{i j l}
\end{aligned}
$$

where matrix $\mathbf{A} \in \mathbb{R}^{n_{N} \times n_{M}}$ and tensor $\mathbf{B} \in \mathbb{R}^{n_{N} \times n_{M} \times n_{M}}$ are defined as $\mathbf{A}=$ $\sum_{k}\left[\tilde{\mathbf{x}}^{(k)}\right]_{i}\left[\mathbf{w}^{(k)}\right]_{i}^{2}\left[\mathbf{y}^{(k)}\right]_{j}$ and $\mathbf{B}=\sum_{k}\left[\mathbf{y}^{(k)}\right]_{l}\left[\mathbf{w}^{(k)}\right]_{i}^{2}\left[\mathbf{y}^{(k)}\right]_{j}$. Based on these expressions, $\mathbf{R}_{G R}$ solves

$$
\mathbf{A}_{i j}=\sum_{l}\left[\mathbf{R}_{G R}\right]_{i l} \mathbf{B}_{i j l}
$$

If the weights, $\mathbf{w}$, are the same for each training data set, then $\mathbf{B}$ does not depend on $i$, and (15) can be written in terms of matrices $\mathbf{A}$ and $\mathbf{B}$ as $\mathbf{A}=\mathbf{R}_{G R} \mathbf{B}$; the reconstruction matrix is calculated and $\mathbf{R}_{G R}=\mathbf{A B} \mathbf{B}^{-1}$. The results for this paper are calculated using this expression for uniform $\mathbf{w}$, since the best selection of weights has not been established.

If $\mathbf{w}$ varies for each element, then $\mathbf{R}_{G R}$ is solved using block diagonal matrix based on $\mathbf{B}$ :

$$
\left[\begin{array}{l}
\mathbf{R}_{11} \\
\vdots \\
\mathbf{R}_{1 M} \\
\vdots \\
\mathbf{R}_{N 1} \\
\vdots \\
\mathbf{R}_{N M}
\end{array}\right]=\left[\begin{array}{lllllll}
\mathbf{B}_{111} & \cdots & \mathbf{B}_{11 M} & \cdots & 0 & \cdots & 0 \\
\vdots & \ddots & \vdots & \ddots & \vdots & \ddots & \vdots \\
\mathbf{B}_{1 M 1} & \cdots & \mathbf{B}_{1 M M} & \cdots & 0 & \cdots & 0 \\
\vdots & \ddots & \vdots & \ddots & \vdots & \ddots & \vdots \\
0 & \cdots & 0 & \cdots & \mathbf{B}_{N 11} & \cdots & \mathbf{B}_{N 1 M} \\
\vdots & \ddots & \vdots & \ddots & \vdots & \ddots & \vdots \\
0 & \cdots & 0 & \cdots & \mathbf{B}_{N M 1} & \cdots & \mathbf{B}_{N M M}
\end{array}\right]^{-1}\left[\begin{array}{l}
\mathbf{A}_{11} \\
\vdots \\
\mathbf{A}_{1 M} \\
\vdots \\
\mathbf{A}_{N 1} \\
\vdots \\
\mathbf{A}_{N M}
\end{array}\right]
$$

The calculation of the GREIT reconstruction matrix may be shown to be equivalent to a scaled generalized Tikhonov solution of the form of section 3.2. In order to illustrate this equivalence, consider a GREIT reconstruction algorithm formulated with uniform weighting $\mathbf{W}$ for each sample, $k$. A training set of size $n_{T}$ training conductivity targets, $\mathbf{y}_{t}^{(k)}$, are drawn from a distribution with covariance $\boldsymbol{\Sigma}_{x}$. For this example, we consider $n_{N}$ samples of only the electronic measurement noise, $\mathbf{y}_{n}^{(k)}$, which is modelled as zeromean Gaussian, and samples are drawn from a distribution with covariance $\boldsymbol{\Sigma}_{n}$. The reconstruction matrix $\mathbf{R}$ minimizes the norm

$$
\left\|\left[\tilde{\mathbf{X}}_{t} \mid 0\right]-\mathbf{R}\left[\mathbf{Y}_{t} \mid \mathbf{Y}_{n}\right]\right\|_{\mathbf{W}}^{2}
$$

where $[\cdot \mid \cdot]$ represents horizontal matrix concatenation and $\mathbf{Y}_{t}=\frac{1}{n_{T}}\left[\mathbf{y}_{t}^{(1)} \cdots \mathbf{y}_{t}^{\left(n_{T}\right)}\right]$, $\tilde{\mathbf{X}}_{t}=\frac{1}{n_{T}}\left[\tilde{\mathbf{x}}_{t}^{(1)} \cdots \tilde{\mathbf{x}}_{t}^{\left(n_{T}\right)}\right]$, and $\mathbf{Y}_{n}=\frac{1}{n_{N}}\left[\mathbf{y}_{t}^{(1)} \cdots \mathbf{y}_{t}^{\left(n_{N}\right)}\right]$. In this case, the matrix $\mathbf{R}$ which 
minimizes the norm is

$$
\begin{aligned}
\mathbf{R} & =\left[\tilde{\mathbf{X}}_{t} \mid 0\right]\left[\mathbf{Y}_{t} \mid \mathbf{Y}_{n}\right]^{T}\left(\left[\mathbf{Y}_{t} \mid \mathbf{Y}_{n}\right]\left[\mathbf{Y}_{t} \mid \mathbf{Y}_{n}\right]^{T}\right)^{-1} \\
& =\tilde{\mathbf{X}}_{t} \mathbf{Y}_{t}^{T}\left(\mathbf{Y}_{t} \mathbf{Y}_{t}^{T}+\mathbf{Y}_{n} \mathbf{Y}_{n}^{T}\right)^{-1} \\
& =\tilde{\mathbf{X}}_{t} \mathbf{Y}_{t}^{T}\left(\mathbf{J} \boldsymbol{\Sigma}_{x} \mathbf{J}^{T}+\boldsymbol{\Sigma}_{n}\right)^{-1}
\end{aligned}
$$

where samples $\mathbf{Y}_{t}$ may be approximated from the Jacobian assuming the body is linear for small targets. This formulation has the same generalized Tikhonov regularization term as that of eqn. (12).

\subsection{Selection of training data}

Training data are selected as small, conductivity contrasting targets spread randomly and uniformly in the plane of 3D model between electrodes. Vertical offsets above and below the plane of $0.25 \times$ diameter of medium are allowed.

The computational cost of calculating $\mathbf{R}_{G R}$ does not change dramatically with the size of the training data set. We therefore use a large number of training samples to avoid any training bias. The minimum training set is much smaller, because, since the model is linear, the key requirement is that the range of $\mathbf{R}_{G R}$ be adequately represented, and it is limited to the number of independent measurements, $\frac{1}{2} n_{E} \times n_{V}$ (which, for a 16 electrode system is 104).

\section{Methods: forward models}

Training data for GREIT requires a forward model which maps conductivity contrast targets, $\mathbf{x}_{t}$, to difference measurements, $\mathbf{y}$. Forward models are built using 3D first order tetrahedral finite elements, and solved using preconditioned linear solvers (Polydorides and Lionheart 2002). Finite element models have been developed for four different geometries: male and female adult chests, a neonatal chest, and a cylinder. The cylindrical form may be used for phantom studies, and to image a pig thorax (which has a roughly circular cross section). Clearly, it is possible to design optimized models for a given patient geometry, and this will offer improved reconstructed images, with the added complexity of designing patient specific FEM meshes. Based on our experience with time-difference EIT, we feel that the four models provided offer most of the accuracy of adaptive meshing.

In the design of FEM models of the thorax, the following issues need to be considered.

- Electrode placement: Several configurations of electrode types and placement are currently used. GREIT considers electrodes placed in a plane at a defined chest height perpendicular to the patient long axis. In the most common configuration, Electrode $\# 1$ is placed on the sternum. Subsequent electrodes are placed on subject left, spaced equidistantly around the chest. When viewing the EIT image, electrodes are numbered clockwise from the top centre, and the subject is oriented 
so the feet would point out of the image. Another common configuration places electrodes \#1 and \#16 on either side of the sternum, and rotates the remaining electrodes accordingly.

Electrodes placed at a mid-thoracic level show a good approximation of the global lung behaviour (Yang and Patterson 2007). There are two main strategies to select the electrode level. One practice is to place electrodes at a defined distance (a few centimeters in adults and millimeters in infants) below the nipple line, although this anatomical marker is somewhat variable for some female and larger or older male subjects. The other approach is to place the electrodes at the level of the $6^{\text {th }}-7^{\text {th }}$ intercostal space at the parasternal line (which corresponds approximately to the nipple line). Other vertical placements have been reported: the $10^{\text {th }}$ intercostal space (Coulombe et al 2005), and the upper chest at the $3^{\text {rd }}$ intercostal space to measure lung perfusion (Smit et al 2004). Placing electrodes too low may result in a large contribution of mediastinum or abdominal impedance changes in the center of the EIT image.

- Electrode size: Electrode size varies between applications. Lung EIT applications typically use standard Ag/AgCl ECG electrodes. For applications with custom EIT electrodes, it is common to use larger electrodes, in order to decrease variability in contact impedance. For infants, ECG type electrodes are often trimmed so that they can fit on the small size chest. FEM models of the electrodes on the neonatal thorax must be adapted to account for the changes in contact impedance due to electrode trimming. The FEM models described in this paper use rectangular electrodes, but may be modifed for other electrode geometries.

- FEM model size and refinement: Increasing the density of elements in the FEM model increases the accuracy of simulations. However, the simulation accuracy is limited by the boundary shape accuracy. We recommend that a minimum FEM model size of 25,000 elements be used for simulation of GREIT training sets. The FEM mesh should be refined in the region of the electrodes, since the highest current densitites occur here. Electrode models should use the complete electrode model (Cheng et al 1989). We also stress the importance of using 3D forward models, since 2D models cannot account for the true distribution of current in the body.

- Conductivity simulations: Simulated targets are created at random positions which uniformly fill the medium in the electrode axis, and a vertical range of $\frac{1}{4}$ of the medium diameter. One important parameter is the use of an appropriate reference conductivity distribution $\boldsymbol{\sigma}_{r}$. The most common assumption for time difference EIT is that the $\boldsymbol{\sigma}_{r}$ is a uniform, homogeneous distribution. However, in a patient, the non-conductive lungs mean a smaller part of the stimulated current penetrates the body centre than would be predicted by an homogeneous distribution. The consequence is that lung contrasts are reduced and "pushed" toward the medium centre. We suggest that simulations be made using $\boldsymbol{\sigma}_{r}$ taken from a realistic model of subject conductivity distributions, which models the lower lung conductivity in 
the central region.

\subsection{Adult models}

Adult FEM models were generated from the visible human body dataset (Ackerman, 1998) which provides radiological and photographic images. The photographic images were used to generate the surface models from which the finite element meshes were generated. The surface models were created using AliasStudio (Autodesk) from the photographic images of the thorax spaced $20 \mathrm{~mm}$ apart. The method used is similar to that used to generate models of the human head for EIT of brain function (Tizzard et al 2005). Rectangular electrodes of $20 \times 40 \mathrm{~mm}$ were specified. Fig. 5 shows an example of the B-Spline curves generated for one slice of the male subject.
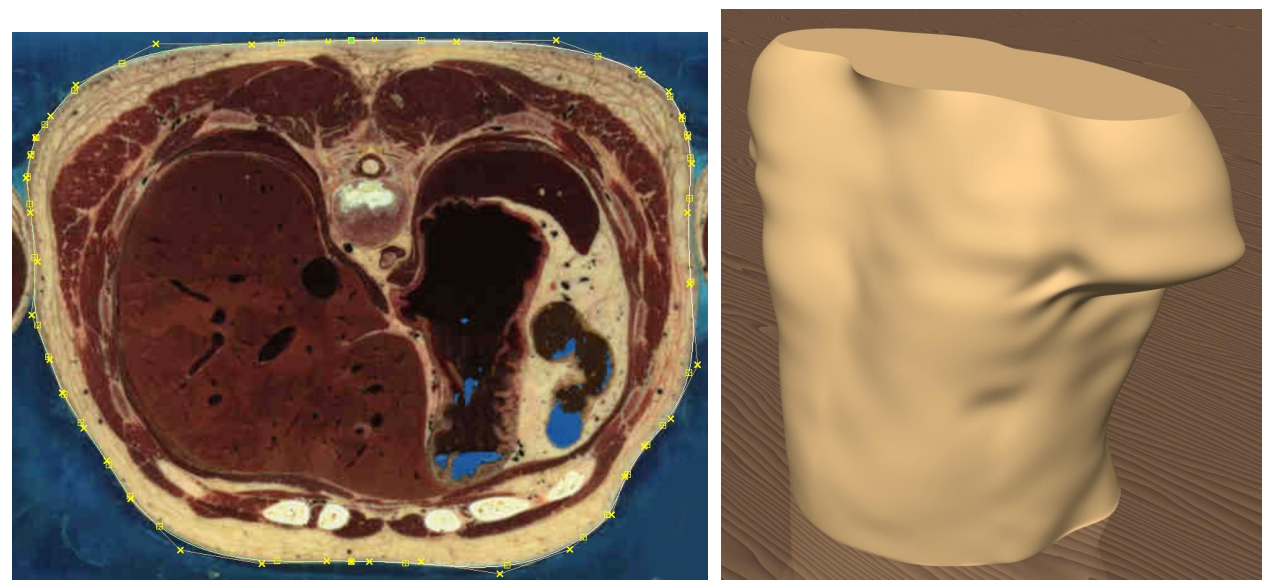

Figure 5. left: One slice of the male photographic visible body dataset showing the modelled B-Spline curves of the boundary. There are two curves, modelling the left and right sides of the boundary, tangentially blended. right: The finished surface model of the male thorax ready for exporting to the FE meshing software.

The final set of curves are used to generate the body surfaces which are then capped top and bottom to form a closed volume. The finished surface model is exported to the mesh-generating software which, in this case, is I-DEAS (Integrated Design Engineering Analysis Software). I-DEAS has the tools to define electrode geometry easily prior to meshing, although any meshing software could be used to achieve this, e.g. NETGEN (Schoberl 1997). The meshes generated in I-DEAS were of similar resolution. The resulting FEM models are shown in Fig. 6. For the male, the model comprised 27,170 elements and 5,548 nodes. For the female, these figures were 27,073 and 5,346 respectively. These data show that for the male, stretch values were between 0.160 and 0.976, with a mean and SD of 0.698 and 0.109 respectively; for the female, stretch values were between 0.198 and 0.982 , with a mean and SD of 0.702 and 0.101 respectively.

The models generated represent a high degree of accuracy of boundary shape to a male and female subject. One limitation is that the source dataset from the visible 
human project was generated from cryogenically frozen cadavers and will therefore be subject to any anatomical inconsistencies arising from the process.
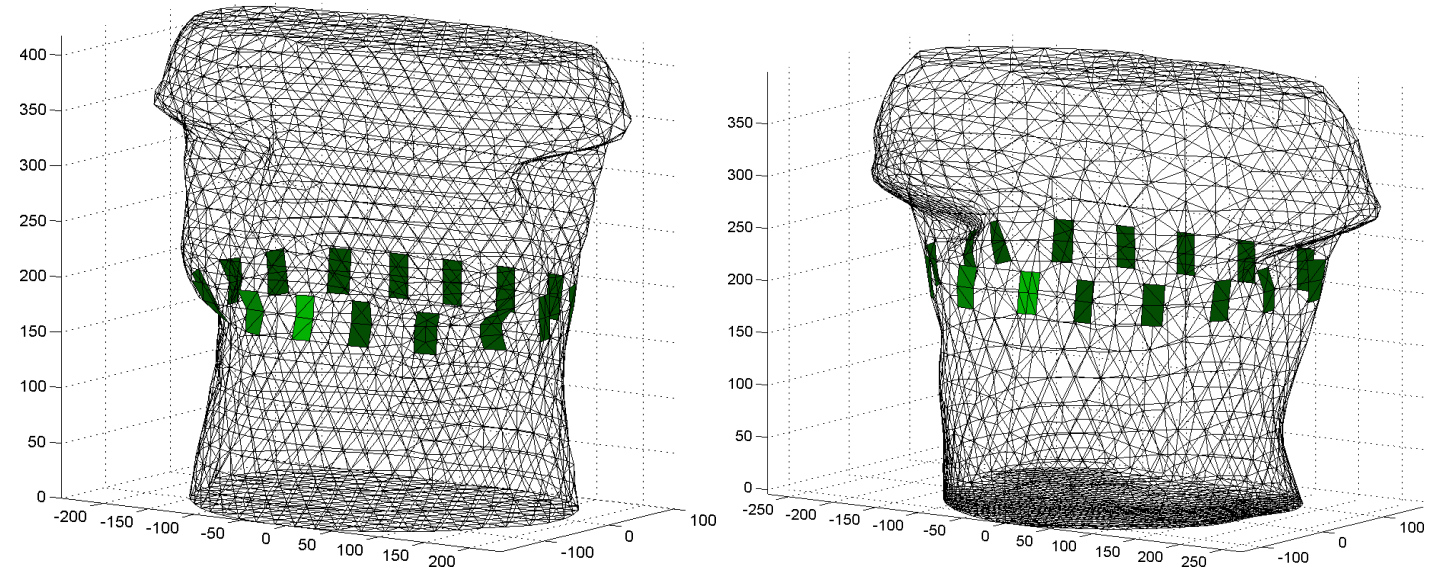

Figure 6. Finite element models of adult thoraces with rectangular electrodes. A wire frame mesh of the surface finite elements is shown. Electrodes are shown in green, with a lighter colour electrode \#1. left: Female subject right: Male subject

\subsection{Neonate models}

The neonatal mesh (Fig. 7) was generated with the surface modelling technique used for the adult models. The images were from X-Ray CT, based on a dataset of an infant with exomphalus. Due to the risks, CT data of healthy infants are not available. The modelling was therefore subject to some assumptions regarding the boundary shape of a healthy subject. Circular electrodes of $8 \mathrm{~mm}$ diameter were specified. The model, which consists of 23,747 elements and 5025 nodes was that used for initial algorithm evaluation in Bayford et al. (2007).

\subsection{Cylindrial models}

A cylindrical FEM model was generated which is suitable for simulations, phantom tank experiments and is also a reasonable model for the thorax shape of common EIT experimental animals, such as pigs. The FEM is generated using NETGEN (Schoberl, 1997). The medium diameter and height are $30 \mathrm{~cm}$ with a maximum element size of $1 \mathrm{~cm}$. Circular electrodes are defined by the intersection of the main cylinder with radial cylinders, and electrode mesh refinement is implemented by specifying a smaller maximum mesh size for each radial cylinder. Fig. 7 shows an image of the resulting mesh (with 124052 tetrahedral elements) as well as the positions of conductivity targets. 

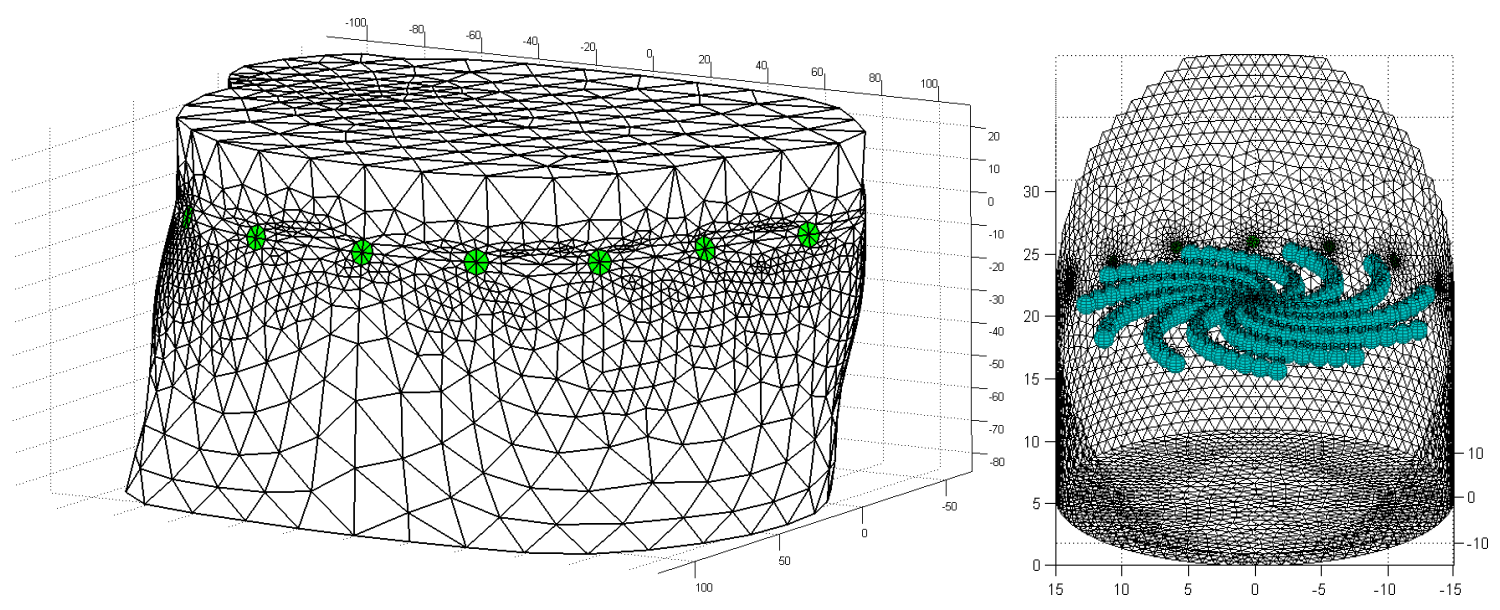

Figure 7. Left: Finite element models of a neonatal thorax with rectangular electrodes. The figure shows a wire frame mesh of the surface finite elements (in green). Right: Cylindrical FEM used for calculation of the main stimulation results of this paper. Image shows boundary elements below a cut plane. The turquoise circles indicate the positions of conductivity targets, $\mathbf{x}_{t}$, used in sec. 5.1.

\section{Evaluation}

Evaluation is performed in two stages. First algorithm performance is evaluated against the figures or merit in section 2. Next, we compare to a representative set of data from simulations and experimental. All evaluation is performed on a 16 electrode EIT configuration using adjacent current stimulation and voltage measurements.

We compare three algorithms for normalized difference EIT: 1) Sheffield Backprojection $\left.\left(\mathbf{R}_{S B P}\right), 2\right)$ Gauss-Newton Reconstruction using a NOSER prior $\left(\mathbf{R}_{G N}\right)$, and 3) GREIT $\left(\mathbf{R}_{G R}\right)$. $\mathbf{R}_{G N}$ is chosen as representative of typical GN difference EIT solutions. In order to standardize noise performance, each algorithm is set to $\mathrm{NF}=0.5$ at the medium centre. This property is inherent to the implemented version of $\mathbf{R}_{S B P}$. For $\mathbf{R}_{G N}, N F$ is set by adjusting the hyperparameter $\lambda$, while for $\mathbf{R}_{G R}$, NF is set by adjusting the weighting $\mathbf{w}^{(k)}$ of noise training data $\mathbf{y}_{n}$. The value of $\lambda$ is chosen using a bisection search.

\subsection{Evaluation: performance figures or merit}

We test each reconstruction matrix, $\mathbf{R}$, for normalized difference EIT against against the figures of merit (sec. 2). Simulation data are generated from the cylindrical phantom as shown in Fig. 7. Five hundred non-conductive targets with a diameter of $5 \%$ of the medium diameter are simulated, in the plane of the electrodes, starting at the medium centre and progressing radially outward. The simulated target is rotated by a fixed angle around the centre for each sample in order to sample the entire FEM geometry.

Fig. 8 shows representative images at three different radial positions. Several effects can be seen from these images. First, Sheffield Backprojection performs well for targets 


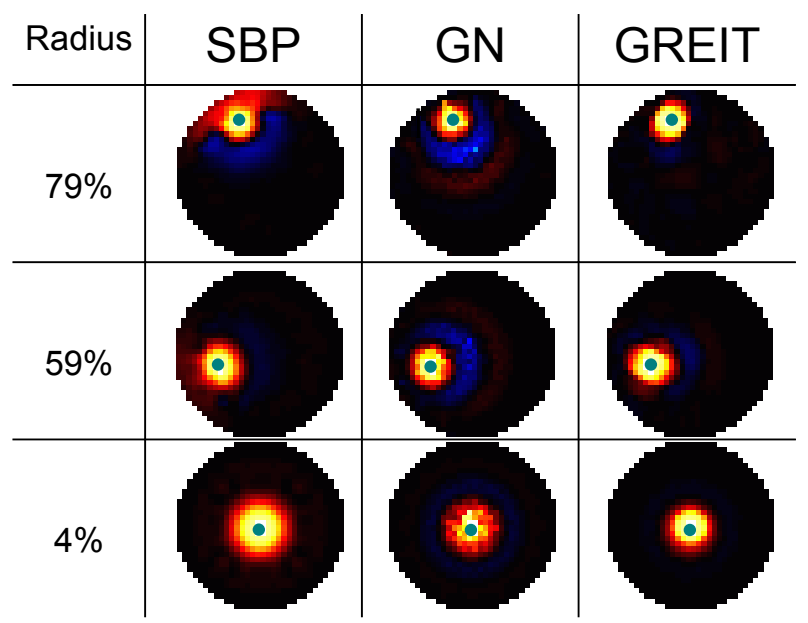

Figure 8. Examples of simulated reconstructed images (red indicates conductive, while blue indicates non-conductive changes) as a function of the simulated target position (left column, in units of radius fraction). Each row shows images reconstructed from the same small conductivity target, $\mathbf{x}_{t}$, shown as a small turquoise circle. left: Sheffield Backprojection $\left(\mathbf{R}_{S B P}\right)$, center: Gauss-Newton $\left(\mathbf{R}_{G N}\right)$, right: GREIT $\left(\mathbf{R}_{G N}\right)$.

that are not too close to the medium boundary; near the boundary, it produces smearing artefacts. GN reconstruction produces clear ringing artefacts, but otherwise shows good performance with good resolution. GREIT gives slightly lower resolution than GN, but gives more constant resolution with very little ringing artefacts, matching the consensus requirements.

Fig. 9 shows algorithm performance for each parameter as a function of radial position. The "noise" that appears on each line is due to the discreteness of the FEM mesh for simulation. There are larger oscillations near the boundary; this reflects variability in performance as the target passes in front of and between the electrodes. Overall, all algorithms perform well in the centre of the medium with poorer performance near the boundary. In comparison to SBP and GN, GREIT shows either an equivalent or an improved ability to meet the desired performance requirements.

\subsection{Evaluation: representative data}

In order to illustrate EIT image reconstructions with these algorithms, we calculate images using $\mathbf{R}_{S B P}, \mathbf{R}_{G N}$, and $\mathbf{R}_{G R}$ for two experiments in which the physiology corresponding to the EIT images is well understood. Due to space constraints, other tests of GREIT are given on the internet at eidors.org/GREIT.

- Recruitment manoeuvre in acute lung injury: These data were measured in an anesthetized ventilated supine piglet as part of the study of Frerichs et al (2003) 

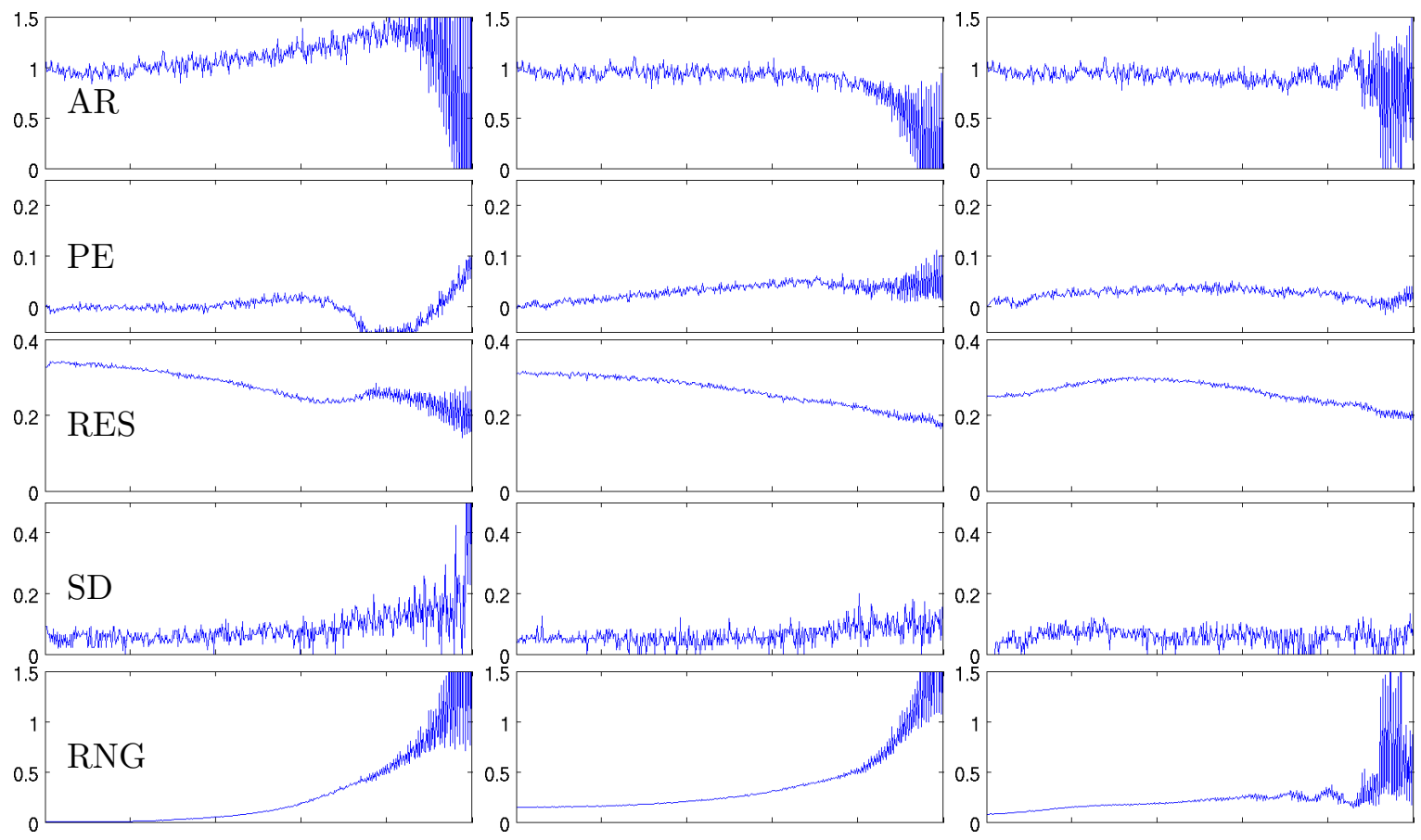

Figure 9. Algorithm performance against simulated tests as a function of radial position. Radial position on each graph is from centre (left) to the body boundary (right). Each column shows results from a different reconstuction algorithm: left: Sheffield Backprojection $\left(\mathbf{R}_{S B P}\right)$, center: Gauss-Newton $\left(\mathbf{R}_{G N}\right)$ right: GREIT $\left(\mathbf{R}_{G R}\right)$ Each row shows a different parameter. From top to bottom: Amplitude Response (AR), Position Error (PE), Resolution (RES), Shape Deformation (SD) and Ringing $(\mathrm{RNG})$

using the Goe MF II system, and performed at the University of Göttingen, Germany. Acute lung injury was created by repeated bronchoalveolar lavage. EIT data were measured during a recruitment manoeuvre during which PEEP was raised and then lowered from 0 to $30 \mathrm{cmH}_{2} \mathrm{O}$ in steps. Images are reconstructed of tidal ventilation at a PEEP of $10 \mathrm{cmH}_{2} \mathrm{O}$ on the inflation and deflation segments of a recruitment manoeuvre. Measurements $\mathbf{v}_{r}$ and $\mathbf{v}$ were calculated from average endexpiratory and end-inspiratory measurements, respectively, at the specified PEEP.

- Regional pneumothorax / pleural effusion: These data were measured in anesthetized ventilated supine pigs as part of the study of Hahn et al (2006) using the Goe MF II EIT system and performed at the University of Göttingen, Germany. Unilateral pleural effusion and pneumothorax was induced by injecting up to $300 \mathrm{ml}$ of air or $300 \mathrm{ml}$ of Ringer solution into the right side via a small incision made in the chest wall and a plastic canula and fixed by a suture and sealed by cyanoacrylate glue. Images are reconstructed of pneumothorax and pleural effusion. Measurement $\mathbf{v}_{r}$ is calculated from an average signal at end-expiration before the intervention, while $\mathbf{v}$ is calculated at end-expiration during injection of air and fluid, respectively. 

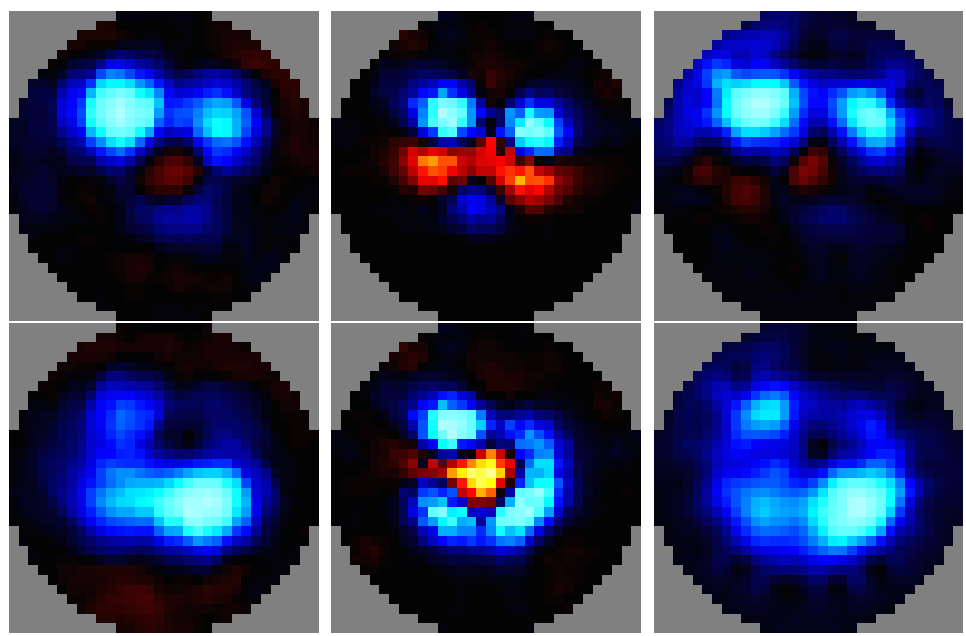

Figure 10. Images of ventilation in a pig with acute lung injury at a PEEP of $10 \mathrm{cmH}_{2} \mathrm{O}$ shown (top row) on the inflation and (bottom row) deflation segment of a stepwise recruitment and derecruitment manoeuvre (data from Frerichs et al 2003). left: GREIT $\left(\mathbf{R}_{G N}\right)$. center: Gauss-Newton $\left(\mathbf{R}_{G N}\right)$, right: Sheffield Backprojection $\left(\mathbf{R}_{S B P}\right)$,
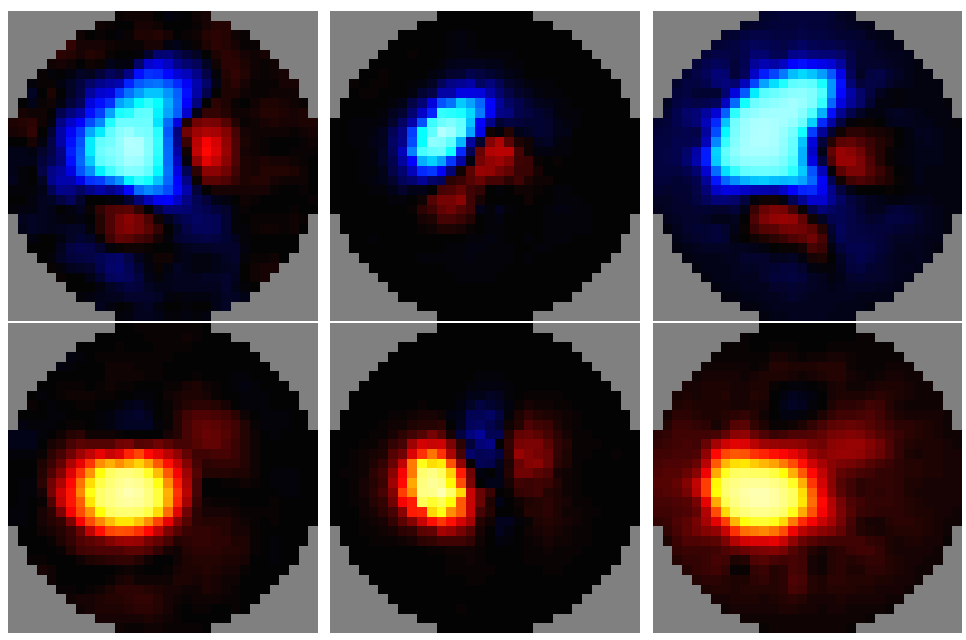

Figure 11. Images of localized (top row) pneumothorax, and (bottom row) pleural effusion in an anesthetized, ventilated pig (data from Hahn et al 2006). left: GREIT $\left(\mathbf{R}_{G N}\right)$. center: Gauss-Newton $\left(\mathbf{R}_{G N}\right)$, right: Sheffield Backprojection $\left(\mathbf{R}_{S B P}\right)$,

\section{Discussion}

This paper describes a unified approach to 2D linear EIT reconstruction of lung images, which we call GREIT. This approach represents a consensus of a large and representative group of experts in EIT algorithm design and clinical applications for pulmonary monitoring. The goal of this work is to address the concern that most clinical and physiological research in lung EIT is done using older and proprietary algorithms, which is an obstacle to interpretation of EIT images because the reconstructed images are not 
well characterized.

The approach consists of: 1) detailed 3D finite element models of the thorax; 2) consensus on the performance figures of merit; and 3) an approach to fit the linear reconstruction matrix, $\mathbf{R}$, to the performance figures. In order of importance, figures of merit are: a) uniform amplitude response, b) small and uniform position error, c) small ringing artefacts, d) uniform resolution, e) limited shape deformation, and f) high resolution. Such figures of merit are to be achieved with small noise amplification and small sensitivity to electrode and boundary movement. The current work leaves several parameters of the algorithm unspecified, including details on the selection of training data and required noise parameters. We are currently undertaking clinical and experimental validation of the GREIT algorithm to establish recommendations for these parameters. It is worth clarifying one disadvantage of the regularized approach presented: Sheffield backprojection tend to map measurement artefacts to "streaks", while GREIT maps the same artefacts to "blobs". It is easier for an operator to notice such streaks, and identify the presence of data errors.

All contributions from this work (algorithms, software, models and test data) have been made available (eidors.org/GREIT) under an open source license which allows gratis commercial and non-commercial use. Software to implement this algorithm is made available under the GNU LGPL (Free Software Foundation, 2007), which requires the source code of any distributed modifications to be made available. FEM models, GREIT reconstruction matrices $\left(\mathbf{R}_{G R}\right)$ and experimental and clinical data provided for evaluation of GREIT, are made available under the Creative Commons Attribution License (Creative Commons, 2007). Users are permitted to copy, distribute, transmit and adapt the works, under the condition of attribution by citing the papers as indicated.

\section{References}

Adler A and Guardo R 1996 Electrical impedance tomography: regularized imaging and contrast detection IEEE Trans. Med. Imaging 15 170-179

Adler A, Guardo R and Berthiaume Y 1996 Impedance imaging of lung ventilation: Do we need to account for chest expansion? IEEE Trans. Biomed. Eng. 43(4) 414-20

Adler A and Lionheart W R B 2006 Uses and abuses of EIDORS: An extensible software base for EIT Physiol Meas 27 S25-S42

Ackerman M J 1998 The Visible Human Project Proc. IEEE 86 504-511

Barber D C and Brown B H 1984 Applied potential tomography J Phys E: Sci Instrum 17 723-733

Barber D C and Brown B H 1988 Errors in reconstruction of resistivity images using a linear reconstruction technique Clin. Phys. Physiol. Meas. 9(suppl. A) 101-4

Barber D C 1989 A review of image reconstruction techniques for electrical impedance tomography Med Phys 16 162-169

Bayford R H Kantartzis P Tizzard A Yerworth R Liatsis P and Demosthenous A 2008 Development of a neonate lung reconstruction algorithm using a wavelet AMG and estimated boundary form Physiol Meas 29 S125-S138.

Brown B H and Seagar A D 1987 The Sheffield data collection system Clin Phys Physiol Meas 8(Suppl A) $91-97$

Brown B W 2003 Electrical impedance tomography (EIT): a review J Medical Eng. $\mathcal{E}$ Technology 27 $97-108$ 
Cheney M, Isaacson D, Newell J C, Simske S and Goble J C 1990 NOSER: an algorithm for solving the inverse conductivity problem Int J Imaging Syst Technol 2 66-75

Cheng KS, Newell JC, Gisser DG, 1989 Electrode Models for Electric Current Computed Tomography IEEE Trans in Biomedical Eng 36 918-924

Cohen-Bacrie C Goussard Y and Guardo R 1997 Regularized Re construction in Electrical Impedance Tomography Using a Variance Uniformization Constraint IEEE Trans Med Imag 16 562-571

Coulombe N, Gagnon H, Marquis F, Skrobik Y, Guardo R, 2005 A parametric model of the relationship between EIT and total lung volume Physiol Meas 26 401-411

Creative Commons/Science Commons 2007 "Creative Commons 3.0 Attribution License" creativecommons.org/licenses/by/3.0/

Frerichs I Dargavillle P A Dudykevych T Rimensberger P M 2003 Electrical Impedance Tomography a method for monitoring regional lung aeration and tidal volume distribution? Intensive Care Med $292312-2316$

GNU Lesser General Public License: Version 3, 29 June 2007 Free Software Foundation, Inc. www.gnu.org/licenses/lgpl.html

Gómez-Laberge C Adler A 2008 Direct EIT Jacobian calculations for conductivity change and electrode movement Physiol Meas 29 S89-S99

Hahn G, Dudykevych T, Frerichs I, Thiel F and Hellige G 2002 A high performance electrical impedance tomography (EIT) system for clinical evaluation studies and space application Proc Conf 2nd European Medical and Biol Eng Vienna, Austria 110-111

Hahn G Just A Dudykevych T Frerichs I Hinz J Quintel M Hellige G 2006 Imaging pathologic pulmonary air and fluid accumulation by functional and absolute EIT Physiol Meas 27 S187-S198

Hahn G Just A Dittmar J Hellige G 2008 Systematic errors of EIT systems determined by easily-scalable resistive phantoms Physiol Meas 29 S163-S172

Hartinger A E Gagnon H Guardo R 2007 Accounting for Hardware Imperfections in EIT Image Reconstruction Algorithms. Physiol Meas 28 13-S27

Lionheart W R B 2004 EIT reconstruction algorithms: pitfalls, challenges and recent developments Physiol Meas 25 125-142

Oh S, Tang T, Sadleir R 2007 Quantitative analysis of shape change in Electrical Impedance Tomography (EIT) in IFMBE Proceedings 17 424-427

Polydorides N and Lionheart W R B 2002 A Matlab toolkit for three-dimensional electrical impedance tomography: A contribution to the Electrical Impedance and Diffuse Optical Reconstruction Software project Meas Sci Technol 13 1871-83

Santosa F Vogelius M 1990 Backprojection algorithm for electrical impedance imaging SIAM J Applied Math 50 216-243.

Schoberl J 1997 NETGEN: An advancing front 2D/3D-mesh generator based on abstract rules Computing and Visualization in Science 1 41-52

Smit H J Vonk Noordegraaf A Marcus R Boonstra A de Vries P M Postmus P E 2004 Determinants of pulmonary perfusion measured by electrical impedance tomography Eur J Appl Physiol 92 45-49

Soleimani M, Gómez-Laberge C and Adler A 2006 Imaging of conductivity changes and electrode movement in EIT Physiol Meas 27 S103-S13

Tizzard A Horesh L Yerworth R J Holder D S Bayford R H 2005 Generating accurate finite element meshes for the forward model of the human head in EIT Physiol Meas 26 S251-61

Vauhkonen M, Vadàsz D, Karjalainen P A, Somersalo E and Kaipio J P 1998 Tikhonov regularization and prior information in electrical impedance tomography IEEE Trans Med Imaging 17 285-93

Victorino JA, Borges JB, Okamoto VN, Matos GF, Tucci MR, Caramez MP, Tanaka H, Sipmann FS, Santos DC, Barbas CS, Carvalho CR, Amato MB 2004 Imbalances in regional lung ventilation: a validation study on electrical impedance tomography. Am J Respir Crit Care Med 169:791-800

Wheeler JL, Wang W, Tang M 2002 A comparison of methods for measurement of spatial resolution in two-dimensional circular EIT images Physiol. Meas. 23 169-176

Wolf GK, Grychtol B, van Genderingen H, Zurakowski D, Thompson JE, Arnold JH 2007. Regional 
lung volume changes in children with acute respiratory distress syndrome during a derecruitment maneuver Crit Care Med 35 1972-1978.

Yang F Patterson R 2007 The contribution of the lungs to thoracic impedance measurements: a simulation study based on a high resolution finite difference model Physiol. Meas. 28 S153-S163

Yorkey T J, Webster J G and Tompkins W J 1987 Comparing reconstruction algorithms for electrical impedance tomography IEEE Trans. Biomed. Eng 34 843-52

Zhang J and Patterson R P 2005 EIT images of ventilation: what contributes to the resistivity changes? Physiol. Meas. 26 S81-S92 